\title{
Avascular Necrosis of the Femoral Head: A Case Report with Emphasis on Imaging Findings
}

\author{
Ansari S ${ }^{1}$, Dhungel K ${ }^{1}$, Ahmad K ${ }^{1}$, Gupta MK ${ }^{1}$, Amanullah MF ${ }^{2}$, Rauniyar RK ${ }^{1}$ \\ ${ }^{1}$ Department of Radiodiagnosis and ${ }^{2}$ Department of Orthopedics, B.P. Koirala Institute of \\ Health Sciences, Dharan, Nepal
}

\begin{abstract}
Avascular necrosis (AVN) of the femoral head is a disease caused by reduced blood supply to the subchondral bone leading to destruction of the hip joint. Most common sites are femoral and humeral head. Initially, patients are asymptomatic, but, in time, AVN leads to joint destruction, requiring surgical treatment and, in later stages, total hip replacement. Magnetic resonance imaging (MRI) has been proved to be a highly accurate method both for early diagnosis and for staging of the disease. We present a case of avascular necrosis of right femoral head in 28 year old male following trauma to hip.
\end{abstract}

Keywords: Avascular necrosis, Osteonecrosis, Femoral head, Radiograph, Magnetic resonance imaging.

\section{Introduction}

Avascular necrosis (AVN) of the femoral head, also known as osteonecrosis is a condition caused by decreased vascular supply to the subchondral bone of the femoral head. ${ }^{1}$ Femoral and humeral heads are the most common sites of involvement. Following ischemic insult, there is death of osteocytes and marrow cells followed by a repair process with osteoclastic resorption of the dead trabeculae and apposition of new bone ${ }^{4}$ and subsequent fracture which results in collapse of the subchondral bone. In

Corrospondence to: Dr. Sajid Ansari, MD, Radiodiagnosis, Department of Radio diagnosis, B.P. Koirala Institute of Health Sciences, Dharan, Nepal.

E mail address: drsajidansari@yahoo.co.in certain cases, genetic factors, such as mutations in the COL2A1 gene, have been associated with the pathogenesis of osteonecrosis. ${ }^{5}$ Males are affected up to three times more than females, and bilateral femoral head osteonecrosis is found in up to

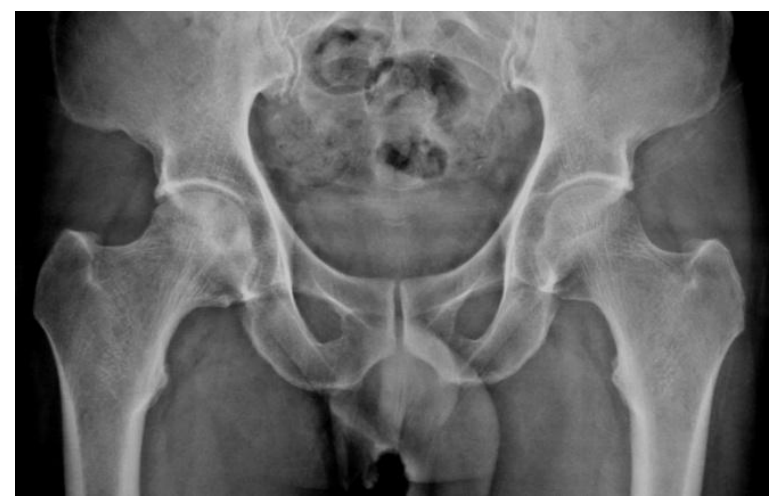

Fig 1: Plain radiograph of hip joint showing sclerosis and subchondral lucency in the right femoral head. 
$75 \%$ of cases. ${ }^{2}$ Patients are usually young adults aged 35 to 45 years.

\section{Case Presentation}

A 28 year old male presented with ongoing intermittent pain in right groin, hip and knee pain for the past 4 months following road traffic accident. Pain was aggravated by walking and relieved by sitting and resting. There was no history of numbness or paresthesias in his lower extremities. No evidence of bowel and bladder dysfunction. The patient did not complain of any night sweats, fever or chills. On physical examination, range of motion of the right hip was limited and painful in all ranges, with most pain being felt in abduction and internal rotation. Plain radiograph of hip joint shows sclerosis and subchondral lucency in the right femoral head (Fig 1). The patient was suspected as having avascular necrosis of the right hip, for which magnetic resonance imaging (MRI) was advised. T1-weighted image (axial section; Fig 2) and T2-weighted image (coronal sections; Fig $3 \mathrm{a}$ and $3 \mathrm{~b}$ ) shows serpiginous, crescent shaped and wedge shaped high signal area with low signal periphery seen in the subchondral region of the right femoral head on the antero-superior aspect. There is mildly irregular contour of right femoral head with mildly reduced joint space. Patient was diagnosed as avascular necrosis of right femoral head.

\section{Discussion}

The common causes of AVN in $75-90 \%$ of cases are chronic steroid use, alcoholism, smoking, hip trauma including femoral neck fractures and hip dislocations, and prior hip surgery. Other etiologies include childhood history of slipped capital femoral epiphysis, caissons disease, systemic lupus erythematosus, vasculitis, sickle cell anemia, coagulopathies, fat embolus syndrome, Gaucher's disease, chemotherapy and/or radiation, organ transplantation, chronic liver disease, etc. $^{2,} 3$ A cause for avascular necrosis is not identified in about $25 \%$ of cases.

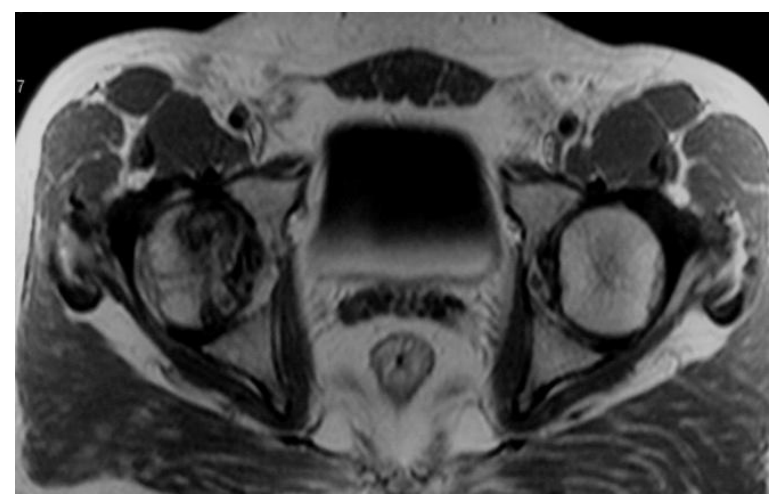

Fig 2: T1-weighted axial MR image showing serpiginous high signal area with low signal periphery in the subchondral region of the right femoral head.

Patients usually present with a combination of hip, groin, thigh or knee pain, usually ongoing for months, with a limited range of motion on examination. Symptoms are generally amplified with weight bearing and relieved with rest. On radiograph, osteopenia will demonstrated in 2 to 5 months. Arlet and Ficat $^{6}$ have developed a staging system of AVN on radiograph. Stage I shows normal radiograph findings, Stage II shows femoral head osteoporosis with areas of cystic lucency and sclerosis, Stage III shows subchondral collapse ("crescent sign"), Stage IV shows segmental progressive femoral head collapse with normal hip joint space and acetabulum and Stage $\mathrm{V}$ shows osteoarthritic changes with joint space narrowing.

MRI has become the imaging modality of choice, as it is highly sensitive and specific 

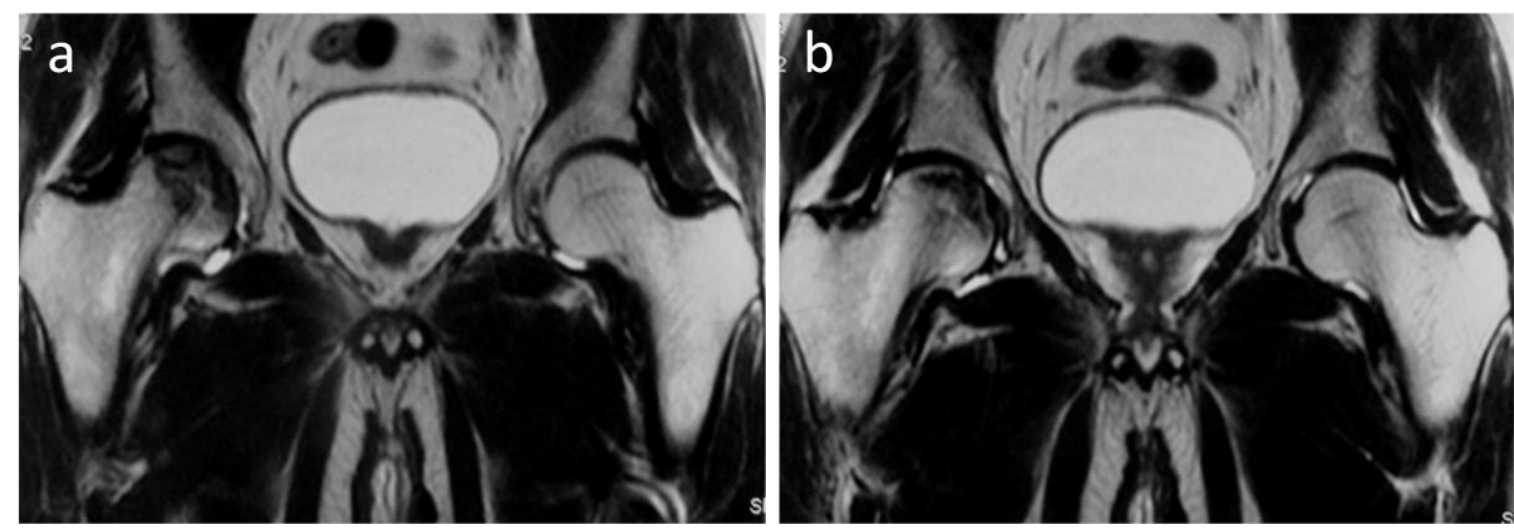

Fig 3a \& 3b: T2-weighted coronal MR images showing wedge shaped (Fig 3a) and crescent shaped (Fig 3b) high signal area with low signal periphery in the subchondral region of the right femoral head. There is mildly irregular contour of right femoral head.

for osteonecrosis, permitting for earlier diagnosis and assessment of articular cartilage. $^{2} \quad$ T1-weighted images shows serpiginous "band-like" lesion with low signal intensity in the anterosuperior femoral head. ${ }^{7}$ The "double-line" sign is seen on T2weighted images and consists of a low signal intensity outer rim and a high signal intensity inner rim. This sign was introduced by Mitchell et al. $^{7}$ and considered pathognomonic for AVN since the outer rim represents the reactive bone and the inner rim the vascular and repair tissue at the necrotic-viable osseous interface. The region within the "double-line" sign may demonstrate hypo-, iso- and hyperintensity relative to the normal marrow.

According to the signals of the central region on T1-weighted and T2-weighted images, a classification scheme was proposed, class A (fat), class B (blood), class C (fluid) and class D (fibrous tissue). ${ }^{7}$ Joint effusions can be seen which are correlated with pain and are commonly found together with bone marrow edema. ${ }^{8}$ Transient osteoporosis can also be seen and is almost always unilateral and the subchondral lesions that might exist are thin and short, probably representing trabecular insufficiency fractures which never proceed to form a circumscribed band. $^{9}$

Radionuclide bone scans are less sensitive and specific than MRI but can be used to detect inflammatory activity in the femoral head when MRI is contraindicated. Reduced uptake may be evident in the blood pool phase in the initial stages, reflecting reduced blood supply. Increased uptake is seen in the later stage reflecting osteoblastic remodeling activity. Computed tomography (CT) is also less sensitive than MRI in detecting osteonecrosis and has a significant radiation burden. Ultrasound may demonstrate synovial swelling and increased joint space in acute stage.

Treatment of AVN includes unloading the hip with non-weight bearing crutches, replacement with bone cement, bone grafting and varus osteotomy. In adults, joint replacement is frequently required in an effort to reduce the probability of developing accelerated degenerative joint disease.

\section{Conclusion}


In AVN, imaging is done to differentiate various causes of painful hip, to confirm a clinically suspected AVN in high risk patients, for staging and treatment planning and response to treatment. MRI has been proved to be a highly accurate method both for early diagnosis and for staging of the disease.

\section{Reference}

1. Li W, Sakai T, Nishii T, et al. Distribution of TRAP-positive cells and expression of HIF-1alpha, VEGF, and FGF-2 in the reparative reaction in patients with osteonecrosis of the femoral head. J Orthop Res. 2009; 27:694-700.

2. Malizos KN, Karantanas AH, Varitimidis $\mathrm{SE}$, et al. Osteonecrosis of the femoral head: etiology, imaging and treatment. Eur J Radiol. 2007; 63:16-28.

3. Mont MA, Zywiel MG, Marker DR, et al. The natural history of untreated asymptomatic osteonecrosis of the femoral head: a systematic literature review. J Bone Joint Surg Am. 2010; 92:2165-2170.

4. Assouline DY, Chang C, Greenspan A, et al. Pathogenesis and natural history of osteonecrosis. Semin

Arthritis

Rheum. 2002; 3 2:94-124.

5. Kannu P, O'Rielly DD, Hyland JC, et al. Avascular necrosis of the femoral head due to a novel $\mathrm{C}$ propeptide mutation in COL2A1. Am J Med Genet A. 2011; 155A:1759-1762.

6. Arlet J, Ficat RP. Dianostic de l'osteonecrose femoro-capitale primitive au stade 1. Rev Chir Orthop 1968; 54:637.

7. Mitchell DG, RaoVM, DalinkaMK, et al. Femoral head avascular necrosis: correlation of MR imaging, radiographic staging, radionuclide imaging, and clinical findings. Radiology 1987; 162:709-15.

8. Huang GS, ChanWP, Chang YC, et al. MR imaging of bone marrow edema and joint effusion in patients with osteonecrosis of the femoral head: relationship to pain. AJR Am J Roentgenol 2003; 181:545-9.

9. Vande Berg BC, Malghem JJ, Lecouvet $\mathrm{FE}$, et al. Idiopathic bone marrow edema lesions of the femoral head: predictive value of MR imaging findings. Radiology 1999; 212:527-35. 